\title{
Even low cTnT levels are indicative of structural heart disease and might be useful in screening
}

\author{
44 hile development of the \\ current generation of \\ troponin assays ... has lead
} to a paradigm shift in the diagnosis of myocardial infarction (MI) and injury, the recently introduced highly sensitive troponin tests will expand the diagnostic potential to the detection of minute injuries of the heart." Hugo Katus' comment comes after the publication of two studies that assessed cardiac troponin T (cTnT) levels in the general population and in elderly individuals, respectively, using a new highly sensitive assay. Professor Katus, from the University of Heidelberg in Germany and who was not involved in either of the studies, believes that "both studies suggest that more-sensitive measurement of troponins may become a new tool in epidemiological studies". He goes on to caution, however, that "whether this new tool will also allow selection of candidates for primary and secondary prevention ... is still illusive".

cTnT measurement is used in the diagnosis of acute MI. However, cTnT levels have also been detected in a small proportion of the general population and have been associated with structural heart disease and adverse cardiovascular (CV) events, including death. James de Lemos and colleagues, therefore, speculated that "if assays for troponin could be developed with increased sensitivity, troponin may represent a powerful tool for population screening". With the development and validation of a highly sensitive cTnT assay in early 2010, these investigators thus set out to assess cTnT levels in a multiethnic cohort representative of the general population.

Blood samples collected from 3,546 individuals aged 30-65 years who enrolled in the Dallas Heart Study in 2000-2002 were assessed in this analysis. cTnT had previously been measured using a standard test (Elecysys-2010 Troponin T; Roche Diagnostics, Indianapolis, IN, USA) with a lower limit of detection of $0.01 \mathrm{ng} / \mathrm{ml}$. For this analysis, cTnT was measured using a highly sensitive assay (Elecysys-2010 Troponin T hs STAT; Roche Diagnostics) with a lower detection limit of $0.003 \mathrm{ng} / \mathrm{ml}$.

By contrast to the standard c'TnT assay, which estimated a prevalence of $0.7 \%$ in the total population, the highly sensitive test demonstrated that $25 \%$ of the general population and $16.2 \%$ of those without $\mathrm{CV}$ disease, chronic kidney disease, subclinical heart disease, diabetes, or hypertension had detectable cTnT levels.

Older participants, men, and black individuals had markedly higher prevalence of detectable cTnT levels than younger participants, women, and white and Hispanic individuals, respectively. The higher the level of cTnT, the higher the prevalence of many other CV risk factors, such as hypertension and diabetes, and of various measures of structural heart disease, including left ventricular hypertrophy. Even in those predicted to be at low risk using the Framingham risk score, detectable cTnT was associated with $\mathrm{CV}$ disease.

Median follow-up was 6.4 years, and detectable cTnT was associated with increased all-cause and CV-disease mortality, in a graded fashion, over this time period. In a fully adjusted model, detectable cTnT levels were independently associated with all-cause mortality.

Although Dr de Lemos notes that the next step is to validate the findings in other data sets, he believes that validation is likely, since a study of elderly individuals in which he was also involved has shown a strong association between low, but detectable, cTnT levels and death and heart failure.

In the study of 4,221 elderly patients (aged $\geq 65$ years) enrolled in the Cardiovascular Health Study and with no prevalent heart failure at baseline, $66.2 \%$ had cTnT levels detectable with the highly sensitive cTnT assay. As seen in the cohort from the Dallas Heart Study, detectable cTnT levels were associated with many CV risk factors as well as various measures of

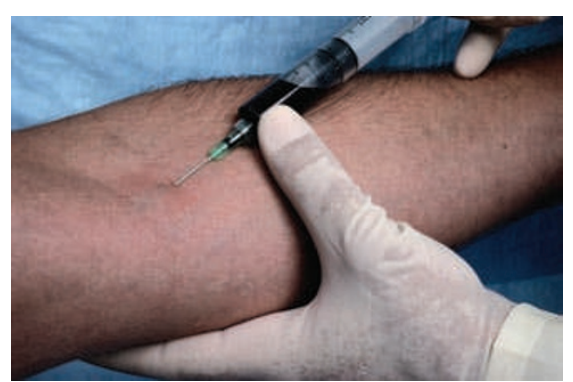

structural heart disease. Over a median follow-up of 11.8 years, higher cTnT levels were associated with higher incidences of new-onset heart failure and CV death. Additionally, change in cTnT levels over the first 2-3 years of follow-up was associated with a shift in risk for both new-onset heart failure and CV death concordant with the direction of change in cTnT levels. Dr de Lemos notes that identification of lifestyle changes and pharmacological therapies that reduce risk in patients with elevated cTnT is an important goal of future research.

"As troponin elevations are not exclusively due to myocardial ischemia," highlights Professor Katus, "clinicians are now confronted with a new challenge to discriminate acute from chronic rise [in cTnT] and MI from myocardial damage not due to acute coronary syndromes." Nevertheless, "cTnT (and also NT-proBNP) may be useful in the office setting to screen for structural heart disease and to augment cardiac risk assessment," comments Dr de Lemos. "These [screening tests] would likely be 'gatekeeper' tests that would prompt additional investigation if abnormal, particularly with cardiac imaging."

Bryony M. Mearns 